\section{Biodegradable Self-Assembling PEG-Copolymer as Vehicle for Poorly Water-Soluble Drugs}

\author{
Louisa Ould-Ouali, ${ }^{1}$ Albertina Ariën, ${ }^{2}$ \\ Joel Rosenblatt, ${ }^{3}$ Aruna Nathan, ${ }^{3}$ Patricia Twaddle, ${ }^{3}$ \\ Tom Matalenas, ${ }^{3}$ Maureen Borgia, ${ }^{3}$ Steve Arnold, ${ }^{3}$ \\ Daniel Leroy, ${ }^{1}$ Mustapha Dinguizli, ${ }^{1}$ \\ Laurence Rouxhet, ${ }^{1,2}$ Marcus Brewster, ${ }^{2}$ and \\ Véronique Préat ${ }^{1,4}$
}

Received June 30, 2003; accepted May 7, 2004

\begin{abstract}
Purpose. To develop self-assembling systems increasing the solubility of poorly water-soluble drugs.

Methods. Low molecular weight liquid biodegradable copolymers were synthesized by ring-opening polymerization using caprolactone (CAP) and trimethylenecarbonate (TMC) as monomers. Various initiators were evaluated. The emulsifying and self-assembling properties were investigated by a water titration method. The selfassembling systems were characterized for size, shape, isotropic behavior, cloud point, surface charge, and critical micellar concentration in order to optimize the polymer synthesis. Finally, the improvement of solubility of model drugs was assessed.

Results. Only diblock monomethyl ether PEG-CAP/TMC copolymers synthesized with monomethyl ether polyethyleneglycol 550 to 2000 as initiator have shown self-assembling properties: upon dilution, these copolymers formed an isotropically clear solution with droplet sizes in the range of 20 to $100 \mathrm{~nm}$. The hypothesis that these diblock polymers form micelles was confirmed by their low critical micellar concentration $\left(10^{-5} \mathrm{~g} / \mathrm{ml}\right)$. The copolymers initated with mmePEG750 had a higher cloud point and better colloidal stability than those initiated with mmePEG 550. The solubility of the poorly water-soluble drugs was increased by 1 to 2 orders of magnitude. Good reproducibility was observed from batch to batch.

Conclusions. The polyester diblock copolymer mmePEG750-CAP/ TMC forms spontaneously stable micelles in aqueous medium and increases the solubility of lipophilic drugs. They are very promising vehicles for the oral delivery of poorly water-soluble drugs.
\end{abstract}

KEY WORDS: diblock polymers; polymeric micelles; selfassembling; poorly water-soluble drugs.

\footnotetext{
$\overline{{ }^{1} \text { Université Catholique de Louvain, Department of Pharmaceutical }}$ Technology, Brussels, Belgium.

${ }^{2}$ Johnson \& Johnson, Pharmaceutical Research and Development, Beerse, Belgium.

${ }^{3}$ Johnson \& Johnson, Center for Biomaterials and Advanced Technologies, Somerville, New Jersey, USA.

${ }^{4}$ To whom correspondence should be addressed. (e-mail : preat@ farg.ucl.ac.be)
}

ABBREVIATIONS: CAP, $\varepsilon$-caprolactone; CMC, critical micellar concentration; Cryo-TEM, cryo-transmission electron microscopy; FASSIF, fasted-state simulated intestinal fluid; FESSIF, fed-statesimulated intestinal fluid; mmePEG, monomethyl ether polyethyleneglycol; mmePEG-CAP/TMC, monomethyl ether polyethyleneglycol-poly ( $\varepsilon$-caprolactone/trimethylene carbonate); $\mathrm{O} / \mathrm{W}$, oil in water emulsion; PCS, photon correlation spectroscopy; PEG, polyethyleneglycol; TMC, trimethylene carbonate; W/O water in oil emulsion.

\section{INTRODUCTION}

The advent of high throughput screening has been a boon to research and development in the pharmaceutical industry. These methodologies, however, select for highly lipophilic derivatives given the nature of the screening and, as a result, there is a general trend for new drug candidates to be poorly water-soluble, of high molecular weight, and to possess poor oral bioavailability (1). The formulation of such compounds into systems that allow for the absorption of the drugs represents, therefore, a real challenge to the formulator. Numerous approaches have been suggested to improve the oral bioavailability of poorly water-soluble drug candidates including micronization (particle size reduction), complexation, formation of a solid solution, preparation of amorphous solid oral dosage forms, lipid based systems (microemulsions, emulsions, liposomes), surfactant and polymer micelles, polymer-based systems (microparticles, microcapsules, etc.), and many others (2-8).

Surfactant micelles are interesting systems as they form spontaneously upon dilution in an aqueous medium. Surfactant molecules possess a hydrophilic and a hydrophobic part, which enables the molecule to adopt different types of organization depending on the surfactant concentration and the properties of the dissolution medium. In general, a surfactant solution consists of three components in dynamic equilibrium: surfactant monomers, micellar aggregates and monomers absorbed as a film at the interfaces (9). In an aqueous medium, above a given concentration called critical micellar concentration (CMC), the surfactant molecules self-assemble with minimal contact between the hydrophobic domain and water, allowing the solubilization of hydrophobic compounds in the core.

Besides conventional surfactants, which have extensively been used for micellar solubilization of drugs, amphiphilic block copolymers can also form micelles and be used as carriers for hydrophobic drugs. In the aqueous environment, the hydrophobic moiety of the ABA block copolymers (e.g., Pluronics: poly(ethylene oxide)-poly(propylene oxide)poly(ethylene oxide)) or AB block copolymers (e.g., poly(ethylene oxide)-poly( $\beta$-benzyl-L-aspartate), poly(ethylene oxide)-poly(DL-lactic-coglycolic acid), poly(ethylene oxide)poly( $\varepsilon$-caprolactone)) forms the core of the micelle while the hydrophilic block poly(ethylene oxide) forms the stabilizing corona. Polymeric micelles have been intensively investigated as parenteral drug vehicles. However, the incorporation of drugs into diblock polymeric micelles is usually a long and multi-step procedure, which often requires the use of organic solvents (for reviews, see Refs. 2,3,10-14). There are various methods for the incorporation of drugs in the blockcopolymer micelles. If the copolymers are water-soluble and can form micelles upon dilution with water, a solution of the drug in an organic solvent may be mixed with an aliquot of the copolymer solution followed by solvent evaporation. Alternatively, the drug in a solvent can be added dropwise to the micellar solution in water and the drug is then incorporated as the solvent evaporates. The direct dissolution may require working at elevated temperatures. The micellization of copolymers, which do not dissolve easily in water, requires the use of the dialysis method. The dialysis method involves different steps: i) dissolution of the copolymer in an organic 
solvent that is miscible with water, ii) dialysis of the solventcopolymer mixture against water. In this case, the drug is first added to the copolymer in the organic solvent (for review, see Ref. 2).

The aim of this study was to develop biodegradable polymers that form self-assembling systems in contact with water. In order to achieve this goal, the polymers will require the following properties: i) The polymer needs to be liquid at room temperature and/or $37^{\circ} \mathrm{C}$ to allow spontaneous selfemulsification without the addition of organic solvents or heat. Hence, low molecular weight random copolymers were identified as possible leads. ii) The polymer must be biodegradable and non-toxic. Novel biodegradable polyesters were therefore synthesized based on monomers which are generally recognized as safe for use as implantable materials such as surgical sutures. The polymers should be self-assembling to avoid the use of surfactants or organic solvents. Hence, the polymers were designed to possess a lipophilic and a hydrophilic moiety.

Based on these criteria, we have developed selfassembling copolymers which form micelles spontaneously and increase the solubility of poorly water-soluble drugs.

\section{MATERIALS AND METHODS}

\section{Chemicals}

The polymers were synthesized by J\&J-Center for Biomaterials and Advanced Technologies (Somerville, NJ, USA). $\varepsilon$-Caprolactone (CAP) was purchased from Union Carbide (Danbury, CT, USA), and trimethylene carbonate (TMC) from Boehringer Ingelheim (Petersburg, VA, USA). 1,3-Propanediol, trimethylol propane, stannous octoate and toluene were from Aldrich (Milwaukee, WI, USA). Polyethyleneglycol with a molecular weight of 600 (PEG 600) and $1000 \mathrm{~g} / \mathrm{mol}$ (PEG 1000) as well as monomethyl ether polyethyleneglycol with a molecular weight of 350, 550, 750 and 2000 $\mathrm{g} / \mathrm{mol}$ (mmePEG 350, mmePEG 550, mmePEG 750 and mmePEG 2000) were purchased from Fluka (Milwaukee, WI, USA). Albumin (fraction V) was purchased from Sigma. All chemical reagents necessary to prepare the different media were purchased from Acros Organics (Pittsburgh, WI, USA). Poloxamer 105 was a gift from ICI surfactants (Cleveland, UK). Pyrene ( $>99 \%$ purity) was purchased from Aldrich. Risperidone, ${ }^{14} \mathrm{C}$-labeled risperidone and ketoconazole were provided by Janssen Pharmaceutica (Beerse, Belgium). Hydrocortisone and indomethacin were purchased from Omega Pharma (Nazareth, Belgium).

\section{Polymer Synthesis and Characterization}

The synthesis of the various "liquid" random copolymers, triblock copolymers and diblock copolymers was performed according to a ring-opening polymerization process (15-17). Preliminary experiments with different monomers had shown that caprolactone and trimethylene carbonate had the best emulsifying and self-assembling properties (data not shown). Hence, three groups of polymers formed from similar ratio of the same monomers: caprolactone (CAP) / trimethylene carbonate (TMC) 50/50 were synthesized and the influence of the initiator used was studied.

\section{Preparation of the Liquid Random Copolymers}

Briefly, the synthesis of CAP/TMC 50/50 polymers (see Table I) was carried out as follows: In a glove box, $50 \mu \mathrm{mol}$ of a stannous octoate solution in toluene, $150 \mathrm{mmol}$ of 1,3propanediol or trimethylol propane, $1.25 \mathrm{~mol}$ of trimethylene carbonate and $1.25 \mathrm{~mol}$ of $\varepsilon$-caprolactone were transferred into a silanized $500 \mathrm{ml}$ single neck round bottom flask equipped with a stainless steel mechanical stirrer and a nitrogen gas blanket. The reaction flask was placed in an oil bath set at $160^{\circ} \mathrm{C}$ for $24 \mathrm{~h}$. The polymer was then devolatilized under vacuum at $90^{\circ} \mathrm{C}$ for 48 to $72 \mathrm{~h}$ (Table I).

\section{Preparation of Triblock Copolymers}

A similar reaction to the synthesis of random copolymer was used to synthesize the triblock copolymers. PEG 600 or PEG 1000 were used as initiators at a monomer/initiator ratio of 12.5/1. (Table I)

\section{Preparation of the PEG Diblock Copolymers}

A similar synthesis procedure was used for the manufacture of PEG diblock copolymers. Monomethyl ether PEG was used as initiator at a monomer/initiator ratio of $13 / 1$ unless stated. Different PEG chain lengths (350, 550, 750, 2000 $\mathrm{g} / \mathrm{mol}$ ) were compared (Table I and Fig. 1).

\section{Characterization of the Polymers Synthesized}

The polymer composition and residual monomer content were analyzed by proton NMR employing a Unity-plus 400 spectrometer from Varian (Palo Alto, CA, USA). Gel permeation chromatography was used to determine the molecular weight and the polydispersity of the polymers. The gel permeation chromatography was performed with a Waters Alliance 2690 system (Milford, MA, USA) equipped with a Wyatt Optilab DSP refractometer, a Dawn multi-angle laser photometer (Wyatt), and Waters Styragel HR 3-4 columns (Table I).

\section{Formulation of Emulsifying and Self-Assembling Systems}

The feasibility of obtaining microemulsions $(18,19)$ with the liquid copolymers as lipophilic phase was evaluated by the construction of ternary phase diagrams (see Fig. 2). The surfactant used was Poloxamer 105. This surfactant was selected because of the presence of a PEG chain, its liquid phase at room temperature and $37^{\circ} \mathrm{C}$ and its cloud point superior to $37^{\circ} \mathrm{C}$. Briefly, 9 binary systems containing various ratios of polymer and surfactant ranging from $10 / 90$ to $90 / 10 \mathrm{w} / \mathrm{w}$ were prepared and equilibrated at $37^{\circ} \mathrm{C}$. Ultrapure water at $37^{\circ} \mathrm{C}$ was then added to the mixture aliquot by aliquot.

The self-assembling properties of the liquid copolymers were assessed by adding water gradually under gentle stirring at $37^{\circ} \mathrm{C}$. The formation of a clear fluid was evaluated visually. Isotropic solutions were also observed under polarized light.

\section{Physicochemical Characterization of the Self-Assembling Systems}

\section{Size and Shape of the Droplets}

The size of the droplets was determined by photon correlation spectroscopy (PCS) using a Coulter N4MD (Coulter 
Table I. Some Properties of CAP/TMC 50/50 mol/mol Copolymers $[ \pm$ Mean $( \pm \mathrm{SD})]$

\begin{tabular}{|c|c|c|c|c|c|c|}
\hline Copolymers* & Initiators $\ddagger$ & Mw§ & Polydispersity§ & $\begin{array}{l}\text { CAP/TMC ratio } \\
\text { in final polymerd }\end{array}$ & $\begin{array}{c}\text { Self-emulsifying } \\
\text { properties }\end{array}$ & $\begin{array}{c}\text { Self-assembling } \\
\text { properties** }\end{array}$ \\
\hline \multicolumn{7}{|l|}{ Random } \\
\hline R1 & 1,3-propanediol & 4567 & 1.6 & $49 \% / 50 \%$ & $\mathrm{~W} / \mathrm{O}$ & No \\
\hline $\mathrm{R} 2$ & trimethylol propane & 4185 & 3.36 & $61 \% / 39 \%$ & $\mathrm{~W} / \mathrm{O}$ & No \\
\hline \multicolumn{7}{|l|}{ Triblock } \\
\hline $\mathrm{T} 1$ & $\begin{array}{l}\text { 1,3-propanediol + } \\
\text { PEG } 600\end{array}$ & 3159 & 1.7 & $51 \% / 48 \%$ & $\mathrm{~W} / \mathrm{O}$ & No \\
\hline $\mathrm{T} 2$ & $\begin{array}{l}\text { 1,3-propanediol + } \\
\text { PEG } 1000\end{array}$ & 3344 & 1.7 & $52 \% / 48 \%$ & $\mathrm{~W} / \mathrm{O}$ & No \\
\hline \multicolumn{7}{|l|}{ Diblock } \\
\hline mmePEG350-CAP/TMC & $\begin{array}{l}\text { Monomethyl ether } \\
\text { PEG } 350\end{array}$ & 4350 & 2.1 & & $\mathrm{O} / \mathrm{W}$ & No \\
\hline $\begin{array}{l}\text { mmePEG550-CAP/TMC } \\
\quad(\mathrm{n}=6) \ddagger\end{array}$ & $\begin{array}{l}\text { Monomethyl ether } \\
\text { PEG } 550\end{array}$ & $4885( \pm 822)$ & $1.8( \pm 0.2)$ & $49.3 \pm 0.4 / 50.3 \pm 0.4$ & $\mathrm{O} / \mathrm{W}$ & Yes \\
\hline $\begin{array}{l}\text { mmePEG750-CAP/TMC } \\
(\mathrm{n}=4) \ddagger\end{array}$ & $\begin{array}{l}\text { Monomethyl ether } \\
\text { PEG } 750\end{array}$ & $5320( \pm 505)$ & $1.9( \pm 0.1)$ & $49.3 \pm 0.4 / 50.1 \pm 0.8$ & $\mathrm{O} / \mathrm{W}$ & Yes \\
\hline mmePEG2000-CAP/TMC & $\begin{array}{l}\text { Monomethyl ether } \\
\text { PEG } 2000\end{array}$ & 6500 & 1.9 & & $\mathrm{O} / \mathrm{W}$ & Yes \\
\hline
\end{tabular}

* The copolymers were synthesized by ring opening polymerization using CAP and TMC as monomers at a molar ratio of 50/50 and stannous octoate as catalyst.

$\dagger \mathrm{n}=$ number of batches synthesized.

$\ddagger$ Initiators were added at a monomer/initiator ratio of $13 / 1$ for the synthesis of the diblock and random copolymers and at a ratio of $12.5 / 1$ for the synthesis of the triblock copolymers.

$\S \mathrm{Mw}$ and polydispersity were measured by GPC.

II The monomer composition was evaluated by NMR.

"Emulsifying properties were determined by water titration with various poloxamer $105 /$ polymer ratios, $\mathrm{W} / \mathrm{O}=$ water in oil microemulsion, $\mathrm{O} / \mathrm{W}=$ oil in water microemulsion.

** Self assembling properties were determined by water titration of the polymer.

Electronics, Beds, UK) or Malvern 4700 (Brookhaven Instruments Corp, Worcestershire, UK) at $25^{\circ} \mathrm{C}$ and at $37^{\circ} \mathrm{C}$. The micelles were also observed by cryo-transmission electron microscopy (cryo-TEM) which allows the observation of the micelles in their wet state and thus avoiding deformations resulting from the drying of the sample occurring in regular TEM sample preparation. Therefore, a $10 \mathrm{mg} / \mathrm{ml}$ aqueous polymer solution was prepared. A small droplet of the sample was placed on a TEM-grid. The excess of solution was eliminated with a filter paper in order to obtain a thin film $(<100$ $\mathrm{nm})$. The sample was then treated with cryogenic liquid ethane. The grid was transferred and mounted under liquid nitrogen on a cryo-TEM holder that was inserted into a TEM Philips CM12. The analyses were performed at $-172^{\circ} \mathrm{C}$, at $120 \mathrm{kV}$.

\section{Surface Charge}

The zeta potential of the micelles was determined with a photon correlation time electrophoretic device (Zetasizer 2000, Malvern, Worcestershire, UK). The analyses were performed on samples diluted in water at different $\mathrm{pHs}(2.45,3.8$, $7.2,9.3)$ or a phosphate buffer $(\mathrm{pH} 6.7,0.05 \mathrm{M})$. The $\mathrm{pH}$ was adjusted with $\mathrm{NaOH}$ or $\mathrm{HCl} 0.1 \mathrm{M}$. The results are the mean of at least 15 measurements.

\section{Surface Tension and Critical Micellar Concentration (CMC) by Tensiometer Method}

The surface tension of the polymers was determined at $37^{\circ} \mathrm{C}$ by the ring method using Du Noüy tensiometer (Krüss, Hamburg, Germany). The reference was ultrapure water.
To investigate if the copolymers form micelles, the surface tension of aqueous solutions containing increasing amounts of mmePEG-CAP/TMC polymer $\left(10^{-3}\right.$ to $\left.10^{-8} \mathrm{~g} / \mathrm{ml}\right)$ was measured. To determine the CMC of the copolymers, the surface tension was plotted against the polymer concentration. The CMC was determined as the intersection of 2 linear regression lines.

\section{Microenvironment and Critical Micellar Concentration by Fluorescence Probe Method}

Three milliliters of a pyrene stock solution $\left(10^{-6} \mathrm{M}\right)$ in acetone was evaporated. Then, $5 \mathrm{ml}$ of an aqueous solution of the copolymer was added to the pyrene. The pyrene concentration was fixed at $6 \times 10^{-7} \mathrm{M}$ for all samples. The polymer concentration varied from 0.1 to $10^{-8} \mathrm{~g} / \mathrm{ml}$. Solutions were placed in a water bath at $70^{\circ} \mathrm{C}$ with stirring for one hour and were then degassed by bubbling oxygen-free nitrogen for 5 min through the solution before recording the spectrum at room temperature (20).

Fluorescence spectra were recorded with a Perkin-Elmer LS50B spectrofluorimeter with a slit smaller than $2.5 \mathrm{~nm}$ (emission). The excitation wavelength corresponded to the maximum intensity obtained ( $\cong 336 \mathrm{~nm})$ in the excitation spectrum. The selected emission wavelengths were the maximum intensities of the first $\left(\mathrm{I}_{1}: 372\right.$ to $\left.373 \mathrm{~nm}\right)$ and third peak $\left(\mathrm{I}_{3}: 383\right.$ to $\left.384 \mathrm{~nm}\right)$ in the emission spectrum. Each spectrum was corrected for scattering caused by the aqueous polymer solution using a blank solution with an identical polymer concentration in absence of pyrene.

To analyze the microenvironment and the $\mathrm{CMC}$ of the 
<smiles>[H][Z9]([H])([H])OCC(C)(C)OC</smiles>

\section{$\left[\mathrm{CH}_{3}\left(\mathrm{CH}_{2}\right)_{3} \mathrm{CH}\left(\mathrm{C}_{2} \mathrm{H}_{5}\right) \mathrm{Co}_{2}\right] \mathrm{Sn}$} in toluene

$\mathrm{CH} 3$

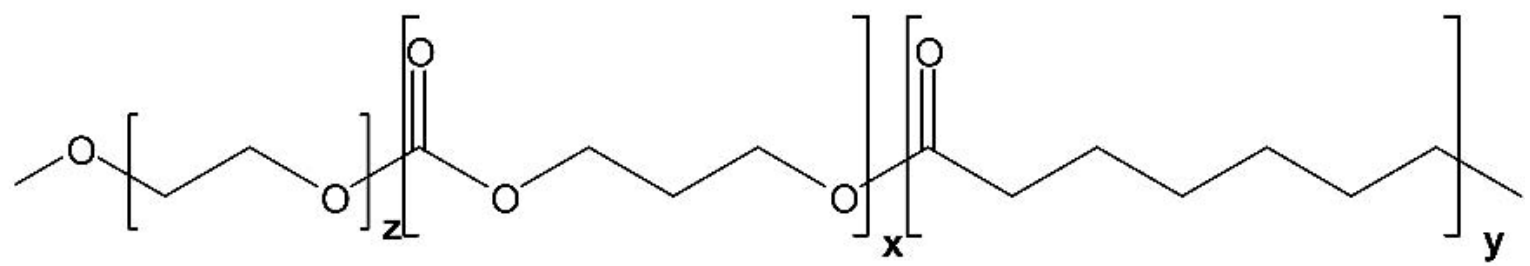

Fig. 1. Chemical reaction schemes of the synthesis of the mme PEG-CAP/TMC copolymers.

polymers, the ratio $I_{1} / I_{3}$ was determined in triplicate. The emission spectrum and hence the $\mathrm{I}_{1} / \mathrm{I}_{3}$ ratio of pyrene depends strongly on the polarity of the environment surrounding the probe molecules. This covers a wide range of polarity from water $\left(\mathrm{I}_{1} / \mathrm{I}_{3}=1.9\right)$ to cyclohexane $\left(\mathrm{I}_{1} / \mathrm{I}_{3}=0.6\right)$. This scale was used to evaluate the microenvironment in the micelle core (21).

To determine the $\mathrm{CMC}$, the $\mathrm{I}_{1} / \mathrm{I}_{3}$ values were averaged over 3 values and plotted vs. the polymer concentration. As the polymer concentration in the aqueous solution increased, the $I_{1} / I_{3}$ ratio decreased and then reached a plateau. The $\mathrm{CMC}$ was determined at the intersection of the 2 lines obtained by linear regression.

\section{Micellization Energy}

The micellization energy was calculated from the CMC by the following calculation, for non-ionic micelles (22)

$$
\Delta G_{0}=R T \ln X_{c m c}
$$

where $\mathrm{R}=$ gas constant $=8.3143 \mathrm{~J} / \mathrm{K}$. mole, $\mathrm{T}=$ temperature in ${ }^{\circ} \mathrm{K}, \mathrm{X}_{\mathrm{cmc}}=$ concentration at $\mathrm{CMC}$ in molar fraction, and $\Delta \mathrm{G}_{0}=$ micellization energy in $\mathrm{kJ} /$ mole.

\section{Cloud Point}

Since non-ionic surfactants usually display a cloud point, the cloud point of the polymers was assessed. A solution with $1 \%$ of polymer was heated over time. The cloud point was defined as the temperature at which the solution becomes cloudy i.e., when the solution becomes turbid at $400 \mathrm{~nm}$ or when it looses its clear isotropic appearance $(23,24)$.

\section{Colloidal Stability}

The colloidal stability of the micelles to added electrolytes was studied as described by Riley et al. (25) wherein 0.5 $\mathrm{ml}$ of polymeric solution $\left(10^{-2} \mathrm{~g} / \mathrm{ml}\right)$ was added to $2.5 \mathrm{ml}$ $\mathrm{Na}_{2} \mathrm{SO}_{4}(0$ to $0.6 \mathrm{M})$. After $15 \mathrm{~min}$ incubation, the turbidity was measured at $400 \mathrm{~nm}$ using a Unicam 8625 Spectrophotometer. The critical flocculation point was determined as the ionic strength where turbidity starts to increase.

\section{Influence of the Dilution Medium}

The influence of the ionic strength, $\mathrm{pH}$ and composition of the dilution medium on the self-assembling properties of the diblock copolymers was analyzed. The following media were tested: i) $\mathrm{NaCl} 9 \%, 0.9 \%, 0.09 \%$, ii) phosphate buffer $0.1 \mathrm{mM} \mathrm{pH} \mathrm{5,} \mathrm{6,} \mathrm{7,} 8$ and 9, iii) $\mathrm{HCl} 0.1 \mathrm{~N}$, iv) albumine 1 and $2 \%, v)$ fasted state-simulated intestinal fluid (FASSIF) (3 $\mathrm{mM}$ sodium taurocholate, $0.75 \mathrm{mM}$ lecithin, $\mathrm{pH} 6.5$, ionic strength $0.15 \mathrm{M}$ ), and vi) fed-state-simulated intestinal fluid (FESSIF) (7.5 mM sodium taurocholate, $3.75 \mathrm{mM}$ lecithin, $\mathrm{pH} 7.5$, ionic strength $0.3 \mathrm{M})$ (26).

\section{Solubilization of Poorly Water-Soluble Drugs}

Since the polymers will be used for the development of new drug delivery systems for poorly water-soluble drugs, the solubility of 4 BCS class II model drugs in the mmePEG550CAP/TMC and mmePEG750-CAP/TMC solutions was assessed. The influence of the lipophilicity and the acidity/ basicity of the drugs on the solubilization properties of the 
A

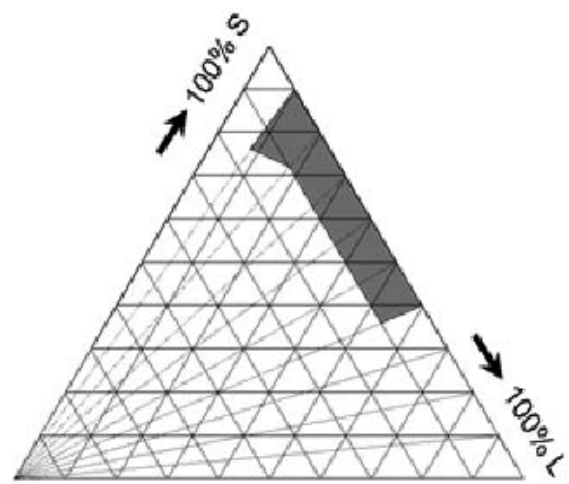

$100 \% \mathrm{~W}$

C

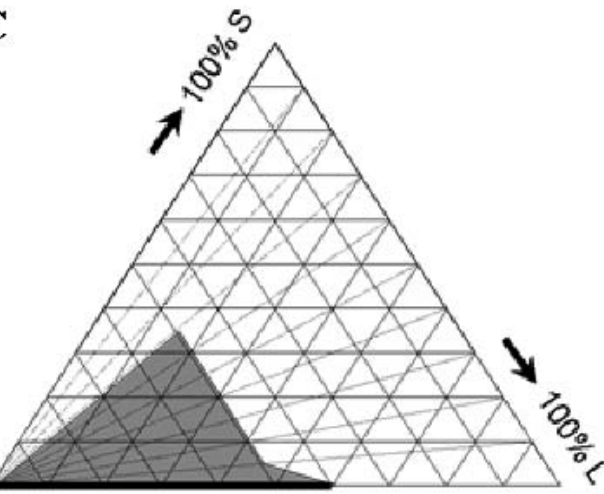

$100 \% \mathrm{~W}$
B

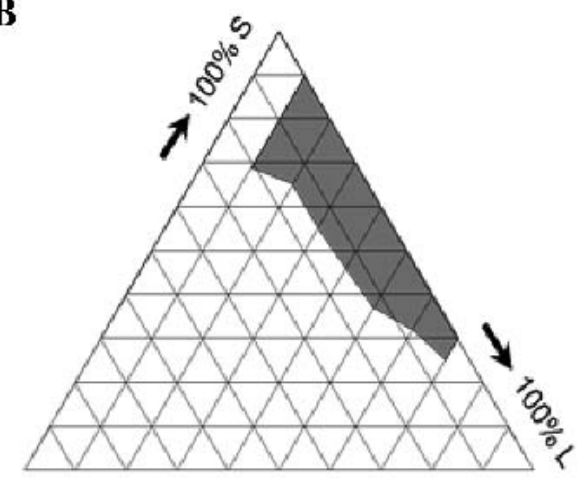

$100 \% \mathrm{~W}$

D

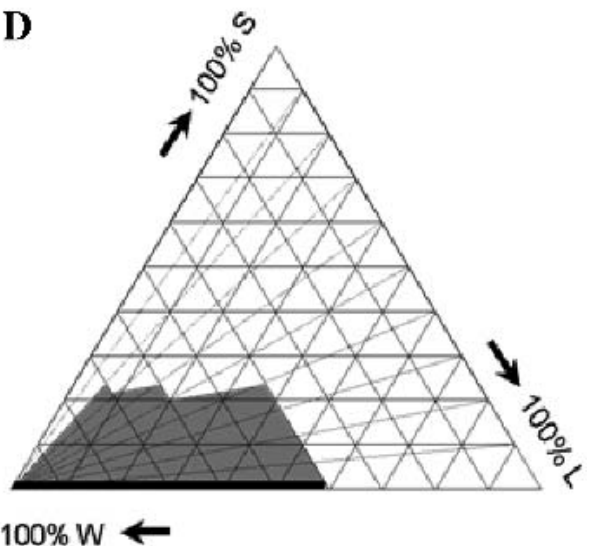

Fig. 2. Ternary phase diagrams obtained by the titration of various mmePEG-CAP/TMC 50/50 copolymer (L)-poloxamer 105 (S) mixtures with water (W). (A) random copolymer R2, (B) triblock copolymer T1, (C) diblock copolymer mmePEG550-CAP/TMC, and (D) diblock copolymer mmePEG750-CAP/ TMC. The experiments were performed at $37^{\circ} \mathrm{C}$. Gray region $=$ clear solution $=$ microemulsion area. Black thick line $=$ self-assembling properties

micelles was evaluated using risperidone, ketoconazole, hydrocortisone and indomethacin as model compounds. The lipophilicity, acidity and solubility of these drugs in water are reported in Table II.

To measure the drug solubility in the polymeric micelles, an excess of drug was mixed with the polymer at room temperature for $24 \mathrm{~h}$ (magnetic stirrer). Water was then added to reach a polymer concentration of $1,3.15,10$ or $31.5 \% \mathrm{w} / \mathrm{v}$.

Table II. Log P, pKa, and Solubility of Risperidone, Ketoconazole, Indomethacin, and Hydrocortisone

\begin{tabular}{lccc}
\hline \multicolumn{1}{c}{ Drug } & Log $\mathrm{P}$ & $\mathrm{pKa}$ & $\begin{array}{c}\text { Solubility at } \mathrm{pH} 7 \\
(\mathrm{~g} / 100 \mathrm{ml})^{*}\end{array}$ \\
\hline Risperidone & 3.04 & $\begin{array}{l}\mathrm{pKa} 1=3.11 \\
\mathrm{pKa} 2=8.24\end{array}$ & $5 \times 10^{-3}$ \\
Ketoconazole & 3.78 & $\begin{array}{l}\mathrm{pKa} 1=3.0 \\
\mathrm{pKa} 2=6.5\end{array}$ & $<1 \times 10^{-3}$ \\
Hydrocortisone & 1.5 & 5.1 & $1.2 \times 10^{-3}$ \\
Indomethacin & 4.3 & 4.5 & $2 \times 10^{-4}$ \\
\hline
\end{tabular}

* The solubility data reported were measured as described in Materials and Methods. The solubility of indomethacin in water was below the detection limit. Therefore, the value reported for this compound is obtained from FDA data.
The drug-polymer-water mixture was stirred for $24 \mathrm{~h}$. The suspension was then filtered through a $0.45-\mu \mathrm{m}$ PVDF filter. The filtered solution was immediately diluted to allow the determination of the drug concentration. The solubility of the drug in water was measured by mixing an excess of the drug with water for $24 \mathrm{~h}$.

The drug concentration in the micelles was determined by UV spectroscopy. Preliminary experiments showed that accurate determinations of the drug concentration in the micelles were possible with this method using as the blank a polymer solution of the same concentration as the sample to be analyzed. Wavelengths of $275 \mathrm{~nm}, 230 \mathrm{~nm}, 278 \mathrm{~nm}$, and 247 $\mathrm{nm}$ were used for risperidone, ketoconazole, indomethacin and hydrocortisone, respectively. The absorbance of the drugs remained constant at various polymer concentrations $(\mathrm{CV}<4 \%)$. The plot of the absorbance vs. the drug concentration in the presence of $10^{-2} \mathrm{~g} / \mathrm{ml}$ of copolymer was linear within the range of drug concentrations tested $\left(\mathrm{R}^{2}>0.99\right)$. The CV was less than $3 \%$. The filtration of the samples containing the polymer and the solubilized drug through $0.45-\mu \mathrm{m}$ PVDF filter (Millipore) induced no significant decrease of the absorbance (less than $1.5 \%$ ). The absorbance of the solutions was measured using a UNICAM 8625, KONTRON Uvikon 940 or Hewlett Packard HP8453 UV/Visible spectrometer. The data are expressed as the mean of three determinations. 
Drug content $(\% \mathrm{w} / \mathrm{w})$ was calculated as follows:

$$
\frac{\text { Mass of drug solubilized }}{\text { Mass of polymer }} \times 100
$$

\section{In vitro Release of Risperidone from the Micelles}

Two hundred fifty microliters of a stock solution of ${ }^{14} \mathrm{C}$ radiolabelled risperidone in ethanol $(10 \mathrm{mg} \mathrm{drug} / \mathrm{ml})$ was placed in vial and the solvent evaporated under a nitrogen flux. $50 \mathrm{mg}$ of the mmePEG750-CAP/TMC polymer was then added and mixed for $24 \mathrm{~h}$ before adding $5 \mathrm{ml}$ of water and filtering through $0.45-\mu \mathrm{m}$ PVDF filter (Millipore). A control experiment to check the release of free risperidone was performed in the same experimental conditions. $1 \mathrm{ml}$ of solution was placed in a $1 \mathrm{ml}$ dialysis bag with a molecular weight cutoff of $1000 \mathrm{Da}$ (SpectroPor Dispodialyzers) and 2 bags were dialyzed against $225 \mathrm{ml}$ of water.

The release of risperidone was conducted at $37^{\circ} \mathrm{C}$. Samples $(5 \mathrm{ml})$ were withdrawn at specific time points for 10 days. The release medium was replaced by $5 \mathrm{ml}$ of water. The radioactivity of the samples was determined with a Liquid Scintillation Counter Wallac 1410 (Pharmacia) using Ultima Gold (Packard Bioscience) as the liquid scintillation analyzer. The cumulative amount of drug released at each sampling point was corrected with the volume of the release medium.

\section{RESULTS}

\section{Optimization of the Synthesis of the Self-Assembling Polymers}

To obtain self-assembling polymers increasing drug solubility, the novel polymers should be liquid, amphiphilic and biodegradable. Monomers and initiators selected to form these polymers were chosen based on these criteria. To be liquid, the polymers had a low molecular weight and were synthesized with 2 monomers. Monomers forming polyester bounds were selected as they can be synthesized by ringopening polymerization and are biodegradable. Preliminary experiments with different monomers including glycolic acid and para-dioxanone showed that the liquid copolymers formed with caprolactone (CAP) and trimethylene carbonate (TMC) had the best emulsifying properties (data not shown). Hence, CAP and TMC were selected as monomers at a molar ratio of 50/50 for the synthesis of lipophilic random copolymers.

According to the type of initiators used, different types of copolymers could be obtained: i) random copolymers when 1,3-propanediol or trimethylolpropane were used as initiator, ii) triblock copolymers with 1,3-propanediol and PEG 600 or
1000 as initiators, iii) diblock copolymers when initiated with mmePEG (see Table I).

The ability of these copolymers to form microemulsions in the presence of a surfactant was first evaluated by adding water to various surfactant/polymer ratios. The random copolymers and the triblock copolymer formed W/O microemulsions whereas the diblock copolymers formed $\mathrm{O} / \mathrm{W} \mathrm{mi-}$ croemulsions (Fig. 2).

As expected, the random copolymers had no selfassembling properties. The triblock copolymers did not form clear solutions upon dilution in water. In contrast, the mmePEG-CAP/TMC diblock copolymers formed spontaneously clear solutions upon dilution in water. This spontaneous self-assembling property was observed with the mmePEG550, mmePEG750 and mmePEG2000 chain length and not with the mmePEG350 (Table I). The mmePEG2000-CAP/TMC polymer is solid at room temperature but after hydration for one hour at room temperature or $37^{\circ} \mathrm{C}$ (addition of 20 to $30 \%$ of water) micelles could be formed.

The monomer/initiator ratio had no influence on the selfassembling properties (data not shown).

\section{Physicochemical Characterization of the Self-Assembling Systems}

As the mmePEG550-CAP/TMC and mmePEG750-CAP/ TMC copolymers form spontaneously clear solutions in water, the physicochemical properties of these self-assembling systems were assessed. Due to their diblock structure with a lipophilic and a hydrophilic moiety and the submicronic size of the self-assembling systems, it was hypothesized that these polymers formed micelles. Hence, their size, zeta potential and $\mathrm{CMC}$ were measured. Their colloidal stability was also assessed.

\section{Size and Shape of the Droplets}

PCS analysis was carried out to determine the size of the self-aggregating structures formed upon the addition of water to the mmePEG-CAP/TMC copolymers. The size of the mmePEG550-CAP/TMC vesicles measured by PCS was 95.4 $\pm 18 \mathrm{~nm}$. The mmePEG750-CAP/TMC copolymers formed droplets with a size in the range of $20 \mathrm{~nm}$. Different batches of mmePEG750-CAP/TMC copolymer gave vesicles with very similar sizes $17 \pm 3.6 \mathrm{~nm}$ (Table III).

Cryo-TEM observations showed that the polymers mmePEG550-CAP/TMC and mmePEG750-CAP/TMC formed spherical structures. Their mean sizes were respectively $10 \pm 3$ and $18 \pm 2 \mathrm{~nm}$ (Fig. 3). Comparison of PCS and cryo-TEM measures suggests that mmePEG550-CAP/TMC micelles could form small aggregates.

Table III. Physicochemical Characterization of Self-Assembling mmePEG550-CAP/TMC and mmePEG750-CAP/TMC Systems

\begin{tabular}{|c|c|c|c|c|c|c|c|}
\hline Polymer & $\begin{array}{c}\text { Size by } \\
\text { PCS (nm) }\end{array}$ & $\begin{array}{c}\text { Cloud point } \\
\left({ }^{\circ} \mathrm{C}\right)\end{array}$ & $\begin{array}{c}\text { CMC ring } \\
\text { method }(\mathrm{g} / \mathrm{ml})\end{array}$ & $\begin{array}{l}\text { CMC pyrene } \\
(\mathrm{g} / \mathrm{ml})\end{array}$ & $\mathrm{I}_{1} / \mathrm{I}_{3}$ & $\begin{array}{c}\text { Micellization } \\
\text { energy } \\
(\mathrm{kJ} / \mathrm{mol})\end{array}$ & $\begin{array}{l}\text { Flocculation } \\
\text { point (ionic } \\
\text { strength M) }\end{array}$ \\
\hline $\begin{array}{l}\text { mmePEG550-CAP/TMC } \\
(\mathrm{n}=5) \\
\text { mmePEG750-CAP/TMC }\end{array}$ & $95.4( \pm 18)$ & 28 & $1.7 \times 10^{-5}\left( \pm 8.3 .10^{-7}\right)$ & $1.5 \times 10^{-5}$ & 1.64 & $-39.1( \pm 3.5)$ & 0.125 \\
\hline$(n=4)$ & $17( \pm 3.6)$ & $53.3( \pm 2.1)$ & $1.2 \times 10^{-5}\left( \pm 1.5 .10^{-6}\right)$ & $3 \times 10^{-5}\left( \pm 1.10^{-5}\right)$ & 1.61 & $-41.1( \pm 5.6)$ & 1.5 \\
\hline
\end{tabular}

For more Information about the polymers, see Table I. [Mean $( \pm \mathrm{SD})]$. 

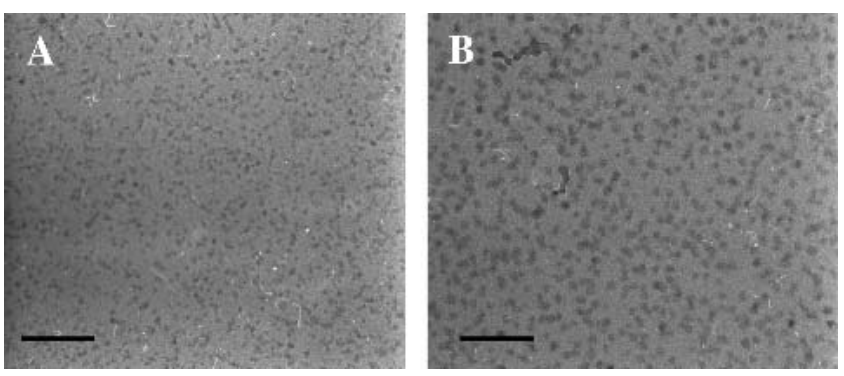

Fig. 3. Cryo-TEM picture of mmePEG550-CAP/TMC and mmePEG750-CAP/TMC polymeric micelles. Scale bar: $100 \mathrm{~nm}$

\section{Surface Charge}

The zeta potential of aqueous solutions of the mmePEG750-CAP/TMC indicated that the polymeric micelles were very slightly charged: the surface charge varied between $-3 \mathrm{mV}$ and $-7 \mathrm{mV}$.

\section{Critical Micellar Concentration (CMC)}

To confirm the hypothesis that the mmePEG-CAP/TMC form micelles, their CMC were determined by two methods, the tensiometer method and the fluorescent probe method.

Upon dilution in water, the surface tension of the mmePEG550-CAP/TMC and mmePEG750-CAP/TMC solutions decreased with increasing polymer concentration until the surface tension remained constant, indicating that a CMC was reached (Fig. 4). The CMC of both copolymers determined by this method, were in a range of $10^{-5} \mathrm{~g} / \mathrm{ml}$ (Table III).

The surface tension of the pure polymers was in the range of $46 \mathrm{mN} / \mathrm{m}$ for all polymers investigated.
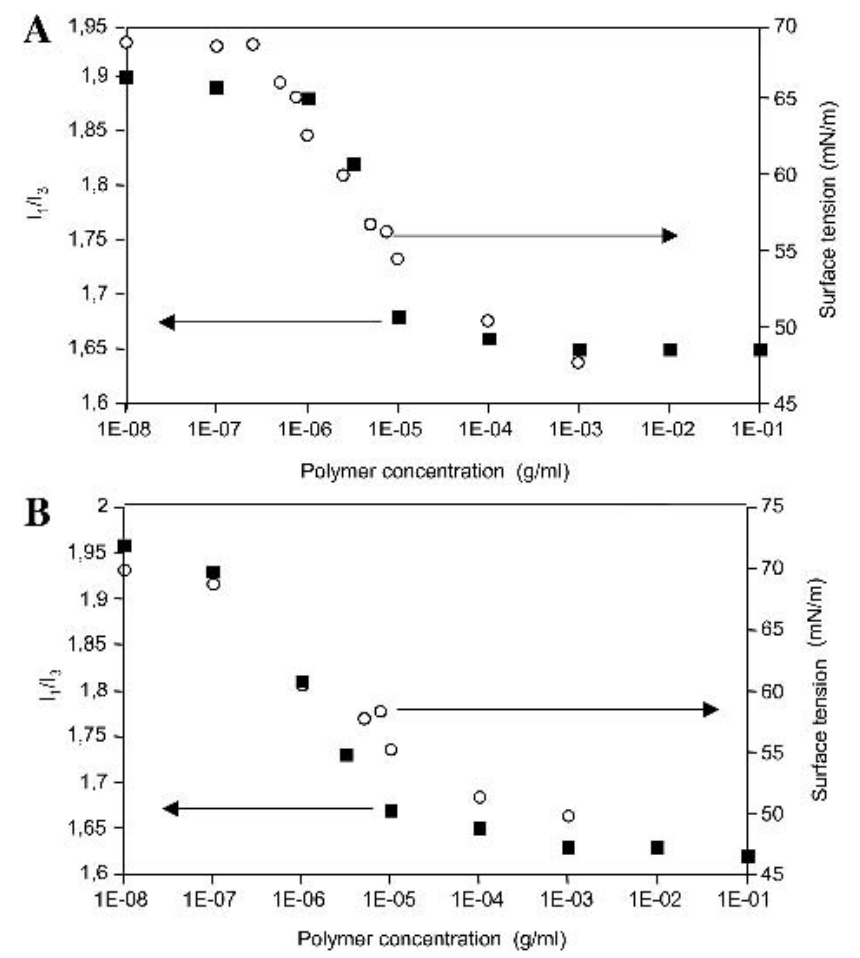

Fig. 4. Surface tension $(O)$ and $I_{1} / I_{3}$ ratio $(\boldsymbol{\square})$ versus copolymer concentration of (A) aqueous solutions of mmePEG550-CAP/TMC and (B) aqueous solutions of mmePEG750-CAP/TMC .
The fluorescence probe was the second method used to determine the $\mathrm{CMC}$ of copolymers. Increasing the concentration of the diblock copolymer mmePEG-CAP/TMC in an aqueous pyrene solution led to several changes in the fluorescence properties. Very small shifts were observed in the excitation (from 335 to $338 \mathrm{~nm}$ ) and emission spectra ( $\mathrm{I}_{1}$. from 372 to $373 \mathrm{~nm}$ and $\mathrm{I}_{3}$ : from 383 to $384 \mathrm{~nm}$ ) of pyrene since the dye molecules moved from a water environment to a more hydrophobic medium $(20,21)$. The $\mathrm{I}_{1} / \mathrm{I}_{3}$ ratio decreased with increasing polymer concentrations to reach a constant base line (Fig. 4). The CMC of the polymers were in a range of $10^{-5}$ $\mathrm{g} / \mathrm{ml}$ (Table III). These results were consistent with those determined by the ring method. Similar CMC values were measured in an aqueous solution $\mathrm{pH} 2$ at $37^{\circ} \mathrm{C}$ (data not shown).

The plots also allowed the determination of the polarity of the micelle core. The $\mathrm{I}_{1} / \mathrm{I}_{3}$ values above the $\mathrm{CMC}$ of the polymer solution indicated that the microenvironment in the micelle core is rather hydrophilic. Indeed the $I_{1} / I_{3}$ values range between 1.64 and 1.61 which corresponds on the pyrene-scale to ethyl acetate (21).

\section{Micellization Energy}

The micellization energy of the different polymers calculated from the CMC is reported in Table III. The negative values of the micellization energy indicate, as expected, that the micellization is spontaneous (it did not require supplementary energy). The difference between the polymers is not significant.

\section{Cloud Point}

As it is the intention to use the proposed systems as a drug delivery system, it is important that the systems remain stable under various temperature conditions. Therefore, the cloud point was determined. The clouding temperatures of mmePEG550-CAP/TMC and mmePEG750-CAP/TMC copolymers were respectively 38 and $58^{\circ} \mathrm{C}$ when evaluated visually and 28 and $53^{\circ} \mathrm{C}$ when evaluated by turbidimetry (Table III), indicating that the cloud point of mmePEG550CAP/TMC at 13/1 monomer/initiator ratio might be too close to the physiologic temperature.

\section{Colloidal Stability}

To check the colloidal stability of the polymeric particles to electrolytes, the critical flocculation point was determined. It was lower for the mmePEG 550-CAP/TMC copolymers than for the mmePEG 750-CAP/TMC copolymers. The flocculation points were respectively 0.1 and $1.5 \mathrm{M}$ ionic strength, indicating that colloidal stability increased with the PEG chain length (Table III and Fig. 5).

\section{Solubility of Poorly Water-Soluble Drugs in the Polymeric Micelles}

The self-assembling polymers were developed to increase the solubility of poorly water-soluble drugs. Therefore, the solubility of 4 BCS class II model drugs (Table II) in aqueous solutions of mmePEG-CAP/TMC polymers was evaluated (Table IV and Fig. 6). Different batches of the 


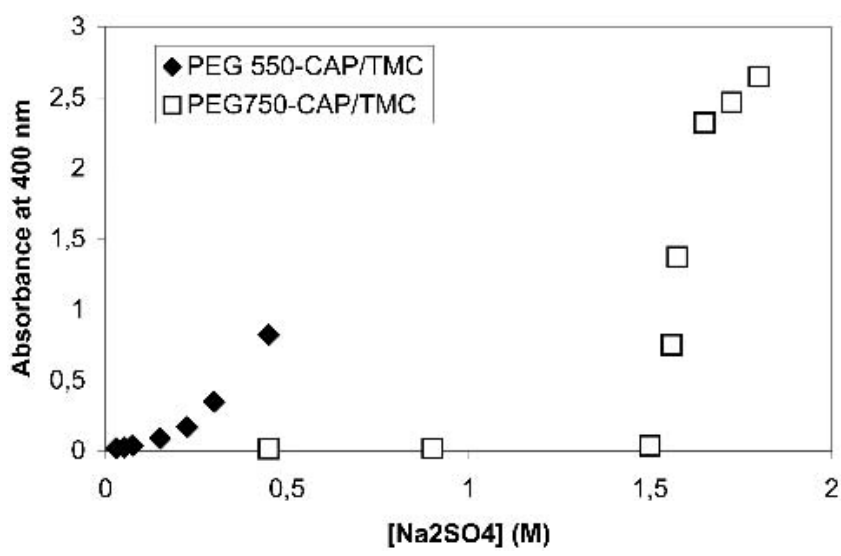

Fig. 5. Turbidity of aqueous solutions of copolymers mmePEG550$\mathrm{CAP} / \mathrm{TMC}$ and mmePEG750-CAP/TMC as function of ionic strength.

mmePEG550-CAP/TMC and of mmePEG750-CAP/TMC were tested for their ability to solubilize these drugs.

The average solubility of risperidone, ketoconazole, hydrocortisone and indomethacin in a solution containing $1 \%$ w/v mmePEG750-CAP/TMC were respectively 33, 17, 43 and $36 \mathrm{mg} / 100 \mathrm{ml}$. Comparison with their solubility in water (respectively $5,<1,1.2$, and $0.2 \mathrm{mg} / 100 \mathrm{ml}$ ) shows that the polymeric micelles can significantly enhance the water-solubility of the model drugs. The drug contents were in the range of $1-4 \% \mathrm{w} / \mathrm{w}$.

Neither the PEG chain length (Table IV) nor the monomer/initiator ratio (data not shown) had an influence on the solubilization properties of the mmePEG-CAP/TMC polymers.

The influence of polymer concentration on the solubility of the model drugs was also assessed. As shown in Fig. 6, the solubility of the drugs increased linearly $\left(\mathrm{R}^{2}\right.$ close to 1$)$ with the polymer concentration ranging from 1 to 10 or $31.5 \% \mathrm{w} / \mathrm{v}$.

\section{In vitro Release of Risperidone from the Micelles}

The stability of the encapsulation of the drug in the micelles was assessed by dialysis equilibrium. As compared to a control with free risperidone, the kinetic of release of risperidone from the micelles was slow: less than $50 \%$ of the drug came out of the mmePEG750-CAP/TMC micelles in 2 days. No significant burst release was observed (Fig. 7).

\section{DISCUSSION}

The aim of this study was to develop novel biodegradable polymers that show self-assembling properties in physiologic conditions and increase the solubilization of poorly watersoluble drugs. Hence, liquid amphiphilic polymers were synthesized by ring opening polymerization using different monomers and initiators. Diblock mmePEG (550 or 750)CAP/TMC 50/50 copolymers with a low molecular weight $(<5000)$ were shown to form, spontaneously, clear solutions in water without requiring heat or solvent. The droplet sizes were in the range of 20 to $100 \mathrm{~nm}$. Moreover, mmePEG2000CAP/TMC 50/50 could also form spontaneously clear solutions in water but the polymer is solid at room temperature and needed to hydrate for one hour at room temperature or $37^{\circ} \mathrm{C}$ (addition of $20 \%$ to $30 \%$ of water). The mixing duration required for the hydration indicates that this polymer would not be an optimal emulsifying polymer to enhance drug solubility.

Due to their amphiphilic diblock structure and to the small size of the droplets, it was very likely that these polymers form micelles in water. To check this hypothesis, two complementary methods were used to assess the CMC. For the diblock copolymers initiated with mmePEG550 or 750, a $\mathrm{CMC}$ could be determined confirming that the mmePEGCAP/TMC copolymers form, spontaneously, micelles in water. The CMC values are in the range of $10^{-5} \mathrm{~g} / \mathrm{ml}$. The CMC values reported in the literature for block copolymers composed of mmePEG or PEG and various polyesters generally range between $10^{-4}$ and $10^{-6} \mathrm{~g} / \mathrm{ml}$ depending on their composition, the ratio between PEG and polyester chain length, their molecular weight $(3,27-33)$. The values are much lower than CMC of low molecular weight surfactants.

Since the micelles will be diluted in vivo, the CMC should be as low as possible to retain the polymers in their micellar form upon dilution and thus retain the drug in the micelles. The CMC of the studied polymers fulfils this requirement: the concentration of the polymers in the gastro-intestinal tract will remain well above the CMC. The mmePEG-CAP/TMC form micelles at different ionic strength, $\mathrm{pH}$ and solution

Table IV. Solubility of Risperidone, Ketoconazole, Hydrocortisone, and Indomethacin $(\mathrm{g} / 100 \mathrm{ml})$ in Aqueous Solutions of the SelfAssembling System Formed by mmePEG-CAP/TMC

\begin{tabular}{|c|c|c|c|c|}
\hline & \multicolumn{4}{|c|}{ Polymer concentration (w/v\%) } \\
\hline & $1 \%$ & $3.15 \%$ & $10 \%$ & $31.5 \%$ \\
\hline \multicolumn{5}{|l|}{ Risperidone } \\
\hline mmePEG550-CAP/TMC & 0.038 & 0.079 & 0.29 & ND \\
\hline mmePEG750-CAP/TMC $(\mathrm{n}=3)$ & $0.03( \pm 0.001)$ & $0.08( \pm 0.013)$ & $0.22( \pm 0.028)$ & $0.64( \pm 0.07)$ \\
\hline \multicolumn{5}{|l|}{ Ketoconazole } \\
\hline mmePEG550-CAP/TMC & 0.022 & 0.055 & 0.22 & 0.65 \\
\hline mmePEG750-CAP/TMC $(\mathrm{n}=2)$ & $0.02( \pm 0.001)$ & $0.06( \pm 0.004)$ & $0.18( \pm 0.005)$ & $0.39( \pm 0.29)$ \\
\hline \multicolumn{5}{|l|}{ Hydrocortisone } \\
\hline mmePEG750-CAP/TMC $(\mathrm{n}=2)$ & $0.04( \pm 0.001)$ & $0.06( \pm 0.006)$ & $0.14( \pm 0.006)$ & ND \\
\hline \multicolumn{5}{|l|}{ Indomethacin } \\
\hline mmePEG750-CAP/TMC & 0.036 & 0.123 & 0.37 & ND \\
\hline
\end{tabular}

The solubility was determined in triplicate at room temperature. For more information about the type of polymers, see Table I. Results are expressed as Mean $( \pm \mathrm{SD})$. ND $=$ not determined. 


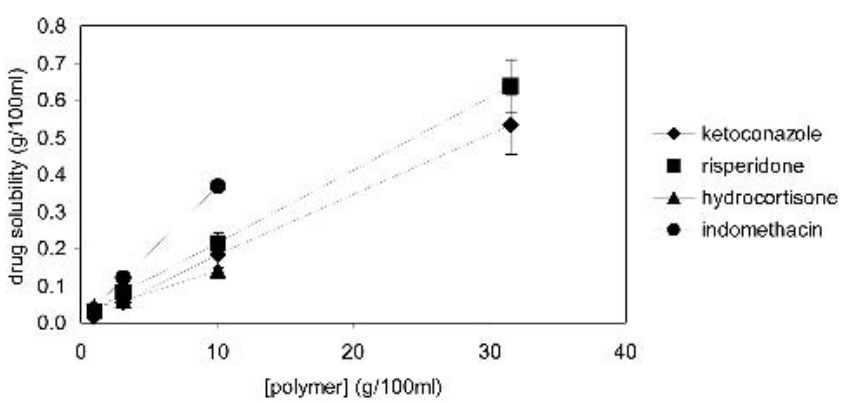

Fig. 6. Solubility $(\mathrm{g} / 100 \mathrm{ml})$ of risperidone, ketoconazole, hydrocortisone, and indomethacin in mmePEG750-CAP/TMC copolymers. The solubility data for ketoconazole are the mean of 2 batches of copolymers. For risperidone the data represent the mean of 3 batches of copolymers while for hydrocortisone the data are the mean of 2 batches. For indomethacin the results of 1 batch are shown. The solubility was determined by UV spectrometry at $25^{\circ} \mathrm{C}$.

composition including simulated gastric and intestinal fluids. Moreover, the drug included in the micelles was slowly released, indicating that the drug will remain in the micelles and will not precipitate out of these micelles. These data indicate that the copolymers could be used for oral delivery of drugs.

As both mmePEG550-CAP/TMC and mmePEG750$\mathrm{CAP} / \mathrm{TMC}$ form, spontaneously, micelles in aqueous media, a thorough characterization was performed to evaluate the effect of the PEG chain length and select the optimal polymer. The length of the CAP/TMC chain remained constant (between 4000 and 4500) The clouding temperature of surfactants and polymers containing PEG groups depends strongly on the poly(ethylene oxide) chain length but is less influenced by the size of the hydrophobic portion of the polymer (24). As shown in Table III, the clouding temperature of the mmePEG-CAP/TMC increased with the PEG chain length. Since the cloud point of mmePEG550-CAP/TMC polymer is close to the physiologic temperature, the copolymer with a PEG chain length of 750 might be more appropriate for pharmaceutical applications. The stability of the colloidal system to the addition of electrolytes was also higher for the mmePEG750-CAP/TMC than for the mmePEG550-CAP/ TMC polymeric micelles. This is in analogy with results reported previously for PEG-poly(lactic acid) or PEG-CAP colloidal systems and for PEGylated liposomes. Comparison of the size of the mmePEG550-CAP/TMC micelles measured by PCS and cryo-TEM also suggests that the steric hindrance by PEG550 could be too low to achieve a good stabilization of the micelles.

To check if the mmePEG-CAP/TMC copolymers could be used as delivery systems for poorly water-soluble drugs, their effect on the solubility of 4 model drugs with different $\log \mathrm{P}$ and $\mathrm{pKa}$ was assessed. The data clearly demonstrate that the polymers enhance the solubility of the 4 drugs in water: the higher the polymer concentration, the higher the solubility. Depending on the physicochemical properties of the drugs, the solubility of the drugs was enhanced up to 180 -fold (for indomethacin) in presence of $1 \% \mathrm{w} / \mathrm{v}$ solution of the polymer. The PEG chain length does not seem to influence the solubilizing properties of the polymer. The drugpolymer compatibility and interactions are the key factors influencing the extent of incorporation of a solubilizate into polymeric micelles. The method of preparation can also affect drug loading efficiency (for review, see Refs. 3,11). Rather low drug content ( $2 \%$ to $4 \% \mathrm{w} / \mathrm{w})$ were achieved compared to the drug content in micelles formed from other PEGpolyesters. For example, indomethacin content in micelles formed from PEG-CAP or PEG-poly(benzyl-L-aspartate) reached respectively $42.2 \%$ (dialysis method) and 20.4 or $22.1 \%$ (dialysis or o/w emulsion method) (32-33). However, the mmePEG750-CAP/TMC micellar solution is several times more effective than hydroxypropyl- $\beta$-cyclodextrin, a well known solubilization enhancer for solubilizing most of the drug tested (data not shown). In addition, the reproducibility of the solubilization properties of the copolymers from one batch to another was excellent.

In the literature, several studies describe the use of PEGcontaining polyesters as micellar carriers of drugs. Mainly biodegradable polyesters consisting of lactide (L or DL), $\varepsilon$-caprolactone, glycolide or copolymers thereof (27-33) or of amino acids (13) are described. To our knowledge, no papers dealing with micellar carriers of mmePEG-CAP/TMC copolymers exists, at the very least concerning the spontaneous formation. In addition, the solubilization of drugs into the micellar carriers described in literature depended largely on the manufacture method used. Generally high entrapment efficiencies could only be achieved when complex methods were used to prepare the drug loaded micelles or nanoparticles. Often the use of organic solvents and/or heat were necessary $(2,3,10,28-33)$. In contrast, the mmePEG750initiated CAP/TMC copolymers described in this paper form spontaneously a clear solution upon dispersion in water and solubilize in an easy way poorly water-soluble drugs.

\section{CONCLUSIONS}

In comparison to existing diblock copolymers which form polymeric micelles, the copolymers composed by mmePEG750-CAP/TMC have the following advantages: i) they form micelles spontaneously in water and in physiologic conditions (no heat or organic solvents are needed to produce micelles and to incorporate drugs), ii) their concentration in the gastro-intestinal tract or blood will remain above the CMC after administration, iii) they enhance the solubility of water soluble drugs.

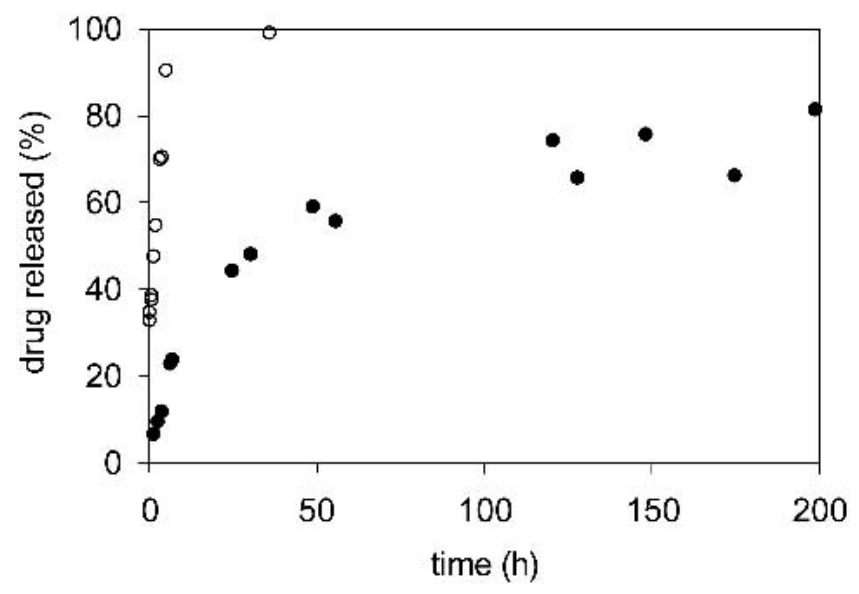

Fig. 7. In vitro release of free risperidone $(\bigcirc)$ and risperidone encapsulated in mme PEG750-CAP/TMC micelles $(\bullet)$. The percentage of risperidone released was measured by dialysis equilibrium at $37^{\circ} \mathrm{C}$. 
Due to their self-assembling properties, they may be administrated orally as "self-microemulsifying drug delivery systems" which will form a clear solution upon agitation in the gastrointestinal tract and enhance the aqueous solubility of the drug solubilized in the polymers. Alternatively, they could also be administered intravenously as micellar solutions.

Diblock mmePEG750-CAP/TMC copolymers seem to be very promising as an interesting drug delivery system for oral delivery of poorly water-soluble drugs.

\section{ACKNOWLEDGMENTS}

The Flemish government, IWT, is gratefully acknowledged for the financial support of this research project. The authors are also grateful to Professor P. Frederik (University of Maastricht, The Netherlands) for his support with the cryoTEM work and Professor J. Demeester (University of Gent, Belgium) for the Zetasizer measurements.

\section{REFERENCES}

1. C. A. Lipinski, F. Lombardo, B. W. Dominy, and P. J. Feeney. Experimental and computational approaches to estimate solubility and permeability in drug discovery and development settings. Adv. Drug Deliv. Rev. 46:3-26 (2001).

2. G. S. Known. Block copolymer micelles as drug delivery systems. Adv. Drug Deliv. Rev. 54:167 (2002).

3. Ch. Allen, D. Maysinger, and A. Eisenberg. Nano-engineering block copolymer aggregates for drug delivery. Colloids Surf. B: Biointerface 16:3-27 (1999).

4. W. N. Charman. Lipids, lipophilic drugs, and oral drug deliverySome emerging concepts. J. Pharm. Sci. 89:967-978 (2000).

5. J. Kreuter. Colloidal drug delivery systems. Drugs and pharmaceutical science, Vol. 66, Marcel Dekker Inc, New York, 1994.

6. M. J. Lawrence and G. D. Rees. Microemulsion based media as novel drug delivery systems. Adv. Drug Deliv. Rev. 45:89-121 (2000).

7. R. H. Muller, K. Mader, and S. Gohla. Solid lipid nanoparticles (SLN) for controlled drug delivery- a review of the state of the art. Eur. J. Pharm. Biopharm. 50:161-177 (2000).

8. A. T. Serajuddin. Solid dispersion of poorly water-soluble drugs: early promises, subsequent problems and recent breakthroughs. J. Pharm. Sci. 88:1058-1066 (1999).

9. D. O. Shah. Micelles, Microemulsions and Monolayers, Marcel Dekker, Inc., New York, 1998.

10. G. S. Kwon and K. Katoaka. Block copolymer micelles as longcirculating drug vehicles. Adv. Drug Deliv. Rev. 16:295-309 (1995).

11. V. P. Torchilin. Structure and design of polymeric surfactantbased drug delivery systems. J. Control. Rel 73:137-172 (2001).

12. A. V. Kabanov, E. V. Batrakova, and V. Y. Alakhov. Pluronic ${ }^{\circledR}$ block copolymers as novel polymer therapeutics for drug and gene delivery. J. Control. Rel 82:189-212 (2002).

13. A. Lavasanifar, J. Samuel, and G. S. Kwon. Poly(ethylene oxide)block-poly(L-amino acid) micelles for drug delivery. Adv. Drug Deliv. Rev. 54:169-190 (2002).

14. V. P. Torchilin. PEG-based micelles as carriers of contrast agents for different imaging modalities. Adv. Drug Deliv. Rev. 54:235252 (2002).

15. R. S. Bezwada, S. C. Arnold, S. W. Shalaby, and B. L. Williams.
Liquid absorbable copolymers for parenteral applications. U.S. Patent 5653 992, 1997 (A).

16. R. S. Bezwada, S. C. Arnold, S. W. Shalaby, and B. L. Williams. Liquid absorbable copolymers for parenteral applications. U.S. Patent 5653 992, 1997 (B).

17. M. Mizutani, S. C. Arnold, and T. Matsuda. Liquid phenylazideend-capped copolymers of caprolactone and trimethylene carbonate: preparation, photocuring characteristics and surface layering. Biomacromolecules 3:668-675 (2002).

18. S. Tenjarla. Microemulsions: an overview and pharmaceutical applications. Crit. Rev. Ther. Drug Carrier Syst. 16:461-521 (1999).

19. I. Danielsson and B. Lindman. The definition of microemulsions. Colloids Surf 3:391-392 (1981).

20. C. L. Zhao and M. A. Winnik. Fluorescence probe techniques used to study micelle formation in water-soluble block copolymers. Langmuir 6:514-516 (1990).

21. T. Nivaggioli, P. Alexandridis, and A. Hatton. Fluorescence probe studies of pluronic copolymer solutions as a function of temperature. Langmuir 11:730-737 (1995).

22. L. J. Chen, S. Y. Lin, and C. C. Huang. Effect of Hydrophobic Chain Length of Surfactants on Enthalpy-Entropy Compensation of Micellization. J. Phys. Chem. B 102:4350-4356 (1998).

23. P. Becher. Encyclopedia of emulsion technology, Marcel Dekker Inc., New York, 1988.

24. B. Jönsson, B. Lindman, K. Holmberg, and B. Kronberg. Surfactants and polymers in aqueous solution. John Wiley \& Sons, New York, 1999.

25. T. Riley, S. Stolnik, C. D. Xiong, M. C. Garnett, L. Illum, and S. S. Davis. Colloidal stability and drug incorporation aspects of micellar-like PLA-PEG nanoparticles. Collö̈ds Surf. B: Biointerfaces. 16:147-159 (1999).

26. E. Galia, E. Nicolaides, D. Horter, R. Lodenberg, C. Reppas, and J. B. Dressman. Evaluation of various dissolution media for predicting in vivo performance of class I and II drugs. Pharm. Res. 15:698-705 (1998)

27. S. A. Hagan, A. G. A. Coombes, M. C. Garnett, L. Illum, S. S. Davis, S. E. Dunn, and M. C. Davies. Polylactide-poly(ethylene glycol) copolymers as drug delivery systems. 1. Characterization of water dispersible micelle-forming systems. Langmuir 12:21532161 (1996).

28. R. T. Liggins and H. M. Burt. Polyether-polyester diblock copolymers for the preparation of paclitaxel loaded polymeric micelle formulations. Adv. Drug Deliv. Rev. 54:191-202 (2002).

29. K. Yasugi, Y. Nagasaki, M. Kato, and K. Kataoka. Preparation and characterization of polymer micelles from poly(ethylene glycol)-poly(D,L-lactide) block copolymers as potential drug carrier. J. Control. Rel 2:89-100 (1999).

30. H. Burt, X. Zhang, P. Toleikis, L. Embree, and W. L. Hunter. Development of copolymers of poly (D,L-Lactide) and methoxypolyethylene glycol as micellar carriers of paclitaxel. Colloids Surf. B Biointerfaces 16:161-171 (1999).

31. X. Zhang, J. K. Jackson, and H. M. Burt. Development of amphiphilic diblock copolymers as micellar carriers of taxol. Int. J. Pharm. 132:195-206 (1996).

32. I. S. Shin, S. Y. Kim, Y. M. Lee, C. S. Cho, and Y. K. Sung. Methoxy poly(ethylene glycol)/ $\varepsilon$-caprolactone amphiphilic block copolymeric micelle containing indomethacin. I Preparation and charcaterisation. J. Control. Rel 51:1-11 (1998).

33. S. B. La, T. Okano, and K. Kataoka. Preparationand characterization of the micelle-forming polymeric drug indomethacinincorporated poly(ethylene oxide)-poly(b-benzyl-aspartate) block copolymer micelle. J. Pharm. Sci. 85:85-90 (1996). 